\title{
The Maximin Principle of $\pi$-Radical Packings
}

\author{
Thomas Devic ${ }^{\ddagger}$, Min Yuan ${ }^{\ddagger}$, Judy Adams ${ }^{\ddagger}$, Daniel C. Fredrickson ${ }^{\ddagger}$, \\ Stephen Lee ${ }^{\ddagger \star}$ and D. Venkataraman ${ }^{\S}$ \\ ${ }^{\ddagger}$ Department of Chemistry and Chemical Biology, \\ Baker Laboratory, Cornell University, Ithaca, NY 14853-1301 \\ ${ }^{\S}$ Department of Chemistry, University of Massachusetts, \\ Amherst, MA 01003
}

Submitted to J. Am. Chem. Soc.

January 18, 2005

*Author to whom correspondence should be addressed (s1137@cornell.edu) 


\section{Supporting Information}

\section{Table of contents:}

- Canonical ring numbering (S2).

- Result of the Cambridge Structural Database searches for molecules containing carbon and nitrogen (S3).

- Crystallographic data and structural descriptions of the magnesium(II) phthalocyanine radical salts $(\mathrm{MgPc})\left(\mathrm{NO}_{3}\right) \cdot 0.5 \mathrm{THF}(\mathbf{1})$ and $(\mathrm{MgPc})\left(\mathrm{ReO}_{4}\right) \cdot 1.5 \mathrm{THF}(2)(\mathrm{S} 8)$.

- TCNQ radical anion packings (S12).

- Complete reference $82(\mathrm{~S} 13)$.

- $\mathrm{x}, \mathrm{y}$ and $\theta$ definitions for fluoranthene and pyrene contour maps (S13). 


\section{Canonical Ring Numbering*.}

Napthalene

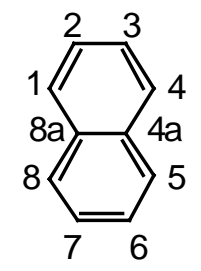

Fluoranthene

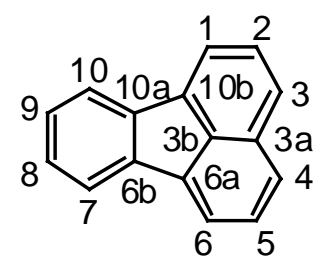

Pyrene

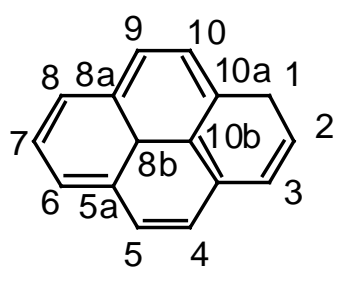

HCCP

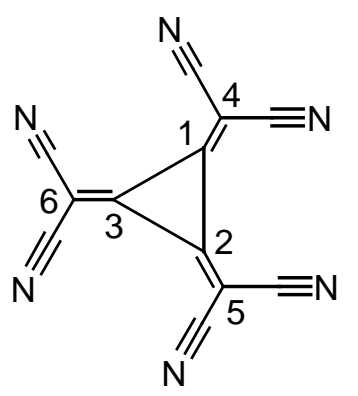

Phthalocyanine

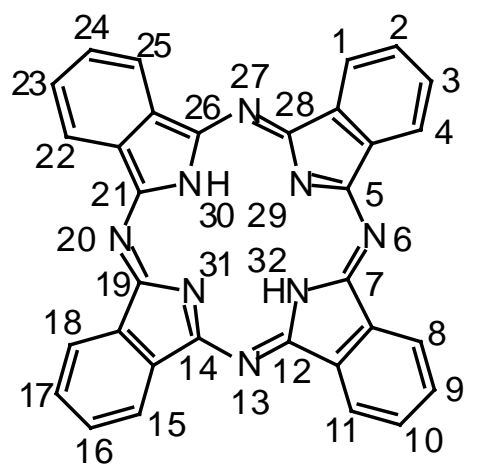

TCNQ

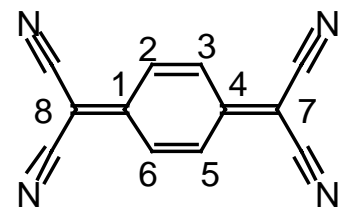

DCNQI

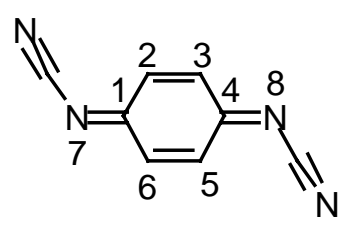

BTCQ

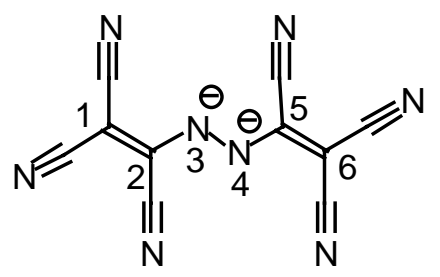

\footnotetext{
* Patterson, A. M.; Capell, L. T.; Walker, D. F. "The Ring Index — A List of Ring Systems Used in Organic Chemistry". American Chemical Society, 1960.
} 


\section{Result of the CSD searches for molecules containing carbon and nitrogen.}

Table SI2 Results of the CSD search on short C,N $\cdots$ C,N contacts $(<3.20 \AA)$. For one particular structure, each line corresponds to a crystallgraphically independent stacking. Structures solved at various temperatures have been counted only once. Only the shortest contact is given. Distances are between carbon atoms except when noticed.

\begin{tabular}{|c|c|c|c|}
\hline Specie & Ref. (CSD) & Dimer geometry & Shortest $\mathrm{C}, \mathrm{N} \cdots \mathrm{C}, \mathrm{N}$ contacts $(<3.20 \dot{A})$ \\
\hline \multirow[t]{2}{*}{$2 H P C$} & MIGCIO & X-form & 3.142 \\
\hline & MIGCUA & X-form & 3.129 \\
\hline \multirow[t]{10}{*}{ MPc } & DIDTEP (Co) & X-form & 3.310 \\
\hline & FANZUP (Ni) & X-form & 3.198 \\
\hline & KARDAI (Ni) & $X$-form & 3.158 \\
\hline & MIGBUZ (Ni) & X-form & 3.169 \\
\hline & MIGCAG $(\mathrm{Cu})$ & $X$-form & 3.125 \\
\hline & MIGCEK (Cu) & X-form & 3.123 \\
\hline & MIGCOU (Ni) & X-form & 3.111 \\
\hline & XOBBAR (Cu) & X-form & 3.091 \\
\hline & $1(\mathrm{Mg})$ & $X$-form & 3.134 \\
\hline & $2(\mathrm{Mg})$ & X-form & 3.129 \\
\hline \multirow[t]{2}{*}{ MPp } & JURFOR (Co) & X-form & 3.169 \\
\hline & SACWAU (Ni) & X-form & 3.199 \\
\hline \multirow[t]{2}{*}{$\mathrm{HCCP}$} & KAHZEY & cross-form & 3.183 \\
\hline & VEYLAM & cross-form & 3.124 \\
\hline \multirow[t]{4}{*}{ TCNQ } & BCRLQM & staggered (short axis) & 3.048 \\
\hline & BEHGEA & staggered (long axis) & 3.167 \\
\hline & BIXHUL & staggered (long axis) & 3.142 \\
\hline & CEHNEI & staggered (long axis) & 3.115 \\
\hline
\end{tabular}




\begin{tabular}{|c|c|c|}
\hline CESCNM10 & staggered (short axis) & 3.191 \\
\hline CURTIS & staggered (long axis) & 3.169 \\
\hline CUZKOX & staggered (long axis) & 3.184 \\
\hline DEMTCN & staggered (long axis) & 3.199 \\
\hline DICLUW & staggered (long axis) & 3.158 \\
\hline DMMTCN01 & staggered (short axis) & 3.191 \\
\hline DUXRET & staggered (long axis) & 3.138 \\
\hline EBPTCQ & staggered (long axis) & 3.110 \\
\hline ECYTCN10 & staggered (long axis) & 3.147 \\
\hline EMTZTQ & staggered (long axis) & 3.098 \\
\hline ENCNQU & staggered (long axis) & 3.143 \\
\hline EPETCQ & staggered (long axis) & 3.195 \\
\hline EPMTCQ & staggered (long axis) & 3.191 \\
\hline ETZCTC & staggered (long axis) & 3.182 \\
\hline FUPJEF & staggered (long axis) & 3.124 \\
\hline GEWCOA & staggered (long axis) & 3.135 \\
\hline HEYCUJ & staggered (long axis) & 3.112 \\
\hline HMBNBQ & staggered (long axis) & 3.172 \\
\hline HUVLIT & staggered (long axis) & 3.167 \\
\hline ICPTCQ & staggered (long axis) & 3.163 \\
\hline IKONOL & staggered (long axis) & 3.158 \\
\hline \multirow[t]{2}{*}{ IKOPAZ } & staggered (long axis) & 3.104 \\
\hline & staggered (long axis) & 3.117 \\
\hline IPONTC & no dimer & $3.064(\mathrm{C} \cdots \mathrm{N}$ between antiparallel $\mathrm{CN})$ \\
\hline JACPUY & staggered (long axis) & 3.169 \\
\hline JANLEP & staggered (long axis) & 3.146 \\
\hline JANLIT & staggered (long axis) & 3.153 \\
\hline
\end{tabular}




\begin{tabular}{|c|c|c|}
\hline JAQQAT & staggered (long axis) & 3.197 \\
\hline JUCMEZ & staggered (short axis) & 3.137 \\
\hline KOBSOJ & staggered (long axis) & 3.156 \\
\hline LILKEW & eclipsed & 3.108 \\
\hline LIWDEA & staggered (long axis) & 3.192 \\
\hline MBITCQ & staggered (long axis) & 3.146 \\
\hline MBPTCQ & staggered (long axis) & 3.310 \\
\hline MBZTCQ & staggered (long axis) & 3.143 \\
\hline MELDEM & staggered (short axis) & 3.195 \\
\hline MELFUE & staggered (long axis) & 3.196 \\
\hline MELGAL & staggered (long axis) & 3.191 \\
\hline MEMTCQ07 & staggered (long axis) & 3.174 \\
\hline MEMTCQ10 & staggered (long axis) & 3.193 \\
\hline MOLNIK & staggered (long axis) & 3.105 \\
\hline MPETCQ & staggered (long axis) & 3.198 \\
\hline MPZTCQ & staggered (long axis) & 3.191 \\
\hline MSTYTQ10 & staggered (long axis) & 3.176 \\
\hline MTCTCN10 & staggered (long axis) & 3.187 \\
\hline NOBKEU & fully staggered & 3.147 \\
\hline NUJVUJ & staggered (long axis) & 3.123 \\
\hline NUJVUJ01 & staggered (short axis) & 3.163 \\
\hline PBPTCQ10 & staggered (long axis) & 3.098 \\
\hline PIFWAC & staggered (long axis) & 3.134 \\
\hline PQTCNQ01 & staggered (long axis) & 3.128 \\
\hline PULWAU & staggered (short axis) & 3.151 \\
\hline PUMWAV & staggered (long axis) & 3.149 \\
\hline PYBUTQ10 & staggered (long axis) & 3.150 \\
\hline
\end{tabular}




\begin{tabular}{|c|c|c|}
\hline \multirow[t]{2}{*}{ PYPTCQ } & staggered (long axis) & 3.139 \\
\hline & staggered (long axis) & 3.173 \\
\hline PYPTCR & staggered (long axis) & 3.194 \\
\hline QMBTCQ & staggered (long axis) & 3.126 \\
\hline RBTCNR01 & staggered (short axis) & 3.137 \\
\hline RBTCNR & staggered (short axis) & 3.189 \\
\hline SEMZOZ & staggered (long axis) & 3.111 \\
\hline SILREK & staggered (long axis) & 3.175 \\
\hline SOZZOW & staggered (short axis) & 3.153 \\
\hline \multirow[t]{2}{*}{ TCQETA02 (40K) } & staggered (long axis) & 3.181 \\
\hline & staggered (long axis) & 3.147 \\
\hline TCQETA06 (110K) & staggered (long axis) & 3.166 \\
\hline TCQETA07 (173K) & staggered (long axis) & 3.199 \\
\hline TTFCQ02 (45K) & staggered (long axis) & 3.165 \\
\hline TTFCQ03 (60K) & staggered (long axis) & 3.176 \\
\hline TTFCQ04 (53K) & staggered (long axis) & 3.162 \\
\hline TTFCQ06 (300K) & staggered (long axis) & 3.181 \\
\hline TTFCQ (100K) & staggered (long axis) & 3.192 \\
\hline \multirow[t]{4}{*}{ TUVGAS } & staggered (long axis) & 3.171 \\
\hline & staggered (long axis) & 3.173 \\
\hline & staggered (long axis) & 3.074 \\
\hline & staggered (long axis) & 3.066 \\
\hline \multirow[t]{3}{*}{ UGENOJ } & staggered (long axis) & 3.195 \\
\hline & staggered (long axis) & 3.063 \\
\hline & staggered (long axis) & 3.063 \\
\hline
\end{tabular}




\begin{tabular}{|c|c|c|c|}
\hline & WOVDEQ & staggered (long axis) & 3.123 \\
\hline & YEYNAR & fully staggered & 3.149 \\
\hline & YOWNON & staggered (short axis) & 3.175 \\
\hline & ZAGCEP & staggered (long axis) & 3.155 \\
\hline DCNQI & TAGZAC & staggered & 3.158 \\
\hline \multirow[t]{2}{*}{$B T C Q$} & REWZEY & staggered & 2.913 \\
\hline & & no dimer & 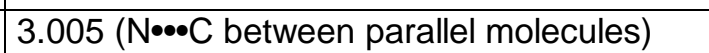 \\
\hline \multirow[t]{16}{*}{ TCNE } & IMEYEE & eclipsed & 2.882 \\
\hline & KAXLEA & eclipsed & 2.905 \\
\hline & KAXLIE & eclipsed & 3.081 \\
\hline & KIYHIJ & eclipsed & 2.988 \\
\hline & KUGHUP & eclipsed & 2.961 \\
\hline & LOCHEQ & eclipsed & 2.925 \\
\hline & LOCHIU & eclipsed & 2.838 \\
\hline & MEFECN & eclipsed & 2.896 \\
\hline & MIKTAB & eclipsed & 2.833 \\
\hline & & eclipsed & 2.933 \\
\hline & & no dimer & $\begin{array}{l}3.022 \quad(\mathrm{~N} \cdots \mathrm{C} \text { between perpendicular } \\
\text { molecules })\end{array}$ \\
\hline & MIKTEF & eclipsed & 2.903 \\
\hline & & no dimer & $\begin{array}{lccc}3.021 & (\mathrm{~N} \cdots \mathrm{C} & \text { between } & \text { perpendicular } \\
\text { molecules }) & & \\
\end{array}$ \\
\hline & ODAFAA & eclipsed & 2.827 \\
\hline & XIDVEL & eclipsed & 2.871 \\
\hline & YAZNUI & eclipsed & 2.920 \\
\hline
\end{tabular}


Crystallographic data and structural descriptions of the magnesium(II) phthalocyanine radical salts.

Table SI1 Crystallographic data and structure refinement for $\mathbf{1}$ and $\mathbf{2}$.

\begin{tabular}{|c|c|c|}
\hline Compound & $(\mathrm{MgPC})\left(\mathrm{NO}_{3}\right) \cdot 0.5 \mathrm{THF}(\mathbf{1})$ & $(\mathrm{MgPC})\left(\mathrm{ReO}_{4}\right) \cdot 1.5 \mathrm{THF}(2)$ \\
\hline empirical formula & $\mathrm{C}_{68} \mathrm{H}_{40} \mathrm{Mg}_{2} \mathrm{~N}_{18} \mathrm{O}_{7}$ & $\mathrm{C}_{76} \mathrm{H}_{56} \mathrm{Mg}_{2} \mathrm{~N}_{16} \mathrm{O}_{11} \mathrm{Re}_{2}$ \\
\hline formula weight & 1269.80 & 1790.41 \\
\hline temperature $[\mathrm{K}]$ & $153(2)$ & $173(2)$ \\
\hline crystal system & orthorhombic & monoclinic \\
\hline space group & $\mathrm{Pca}_{1}$ & $\mathrm{P} 2_{1} / \mathrm{c}$ \\
\hline $\mathrm{a}[\AA ̊]$ & $14.9214(6)$ & $14.1490(11)$ \\
\hline $\mathrm{b}[\AA ̊]$ & $17.0155(7)$ & $30.698(2)$ \\
\hline$c[\AA ̊]$ & $22.5556(8)$ & $15.6929(12)$ \\
\hline \multicolumn{3}{|l|}{$\alpha\left[^{\circ}\right]$} \\
\hline$\beta\left[^{\circ}\right]$ & & $98.634(2)$ \\
\hline \multicolumn{3}{|l|}{$\gamma\left[^{\circ}\right]$} \\
\hline $\mathrm{V}\left[\AA^{3}\right]$ & $5726.8(4)$ & 6738.9(9) \\
\hline Z & 4 & 4 \\
\hline$\rho_{\text {calcd }}\left[\mathrm{g} \cdot \mathrm{cm}^{-3}\right]$ & 1.473 & 1.765 \\
\hline$\mu\left[\mathrm{mm}^{-1}\right]$ & 0.120 & 3.687 \\
\hline$F(000)$ & 2616 & 3543 \\
\hline crystal size $[\mathrm{mm}]$ & $0.20^{*} 0.10^{*} 0.10$ & $0.40^{*} 0.30 * 0.15$ \\
\hline$\theta$ range $\left[^{\circ}\right]$ & $1.81-24.74$ & $1.33-28.33$ \\
\hline reflections collected & 34784 & 57260 \\
\hline
\end{tabular}




\begin{tabular}{lll}
\hline independent reflections & 5030 & 16610 \\
$\mathrm{R}_{\text {int }}$ & 0.1109 & 0.0784 \\
reflections with $\mathrm{I}>2 \sigma(\mathrm{I})$ & 3822 & 11545 \\
parameters & 851 & 964 \\
GOF on $\mathrm{F}^{2}$ & 1.056 & 1.051 \\
$\mathrm{R} 1^{\mathrm{a}}(\mathrm{I}>2 \sigma(\mathrm{I})) / \mathrm{wR}{ }^{\mathrm{b}}$ & $0.0637 / 0.1520$ & $0.0437 / 0.0925$ \\
largest diff. peak/hole $\left[\mathrm{e}^{\AA}-3\right]$ & $0.544 /-0.348$ & $2.475 /-1.428$ \\
\hline${ }^{\mathrm{a}} \mathrm{R} 1=\sum(\|\mathrm{Fo}|-| \mathrm{Fc}\|) / \Sigma|\mathrm{Fo}|{ }^{\mathrm{b}}{ }^{\mathrm{wR} 2}=\left[\Sigma\left(\mathrm{w}\left(\mathrm{Fo}^{2}-\mathrm{Fc}^{2}\right)^{2}\right) / \Sigma\left(\mathrm{Fo}^{2}\right)^{2}\right]^{1 / 2}$.
\end{tabular}

\section{Structural description}

1 and 2 are the first magnesium(II) phthalocyanine salts structurally described.

1 crystallizes in an orthorhombic setting in the non-centrosymmetric Pca2 ${ }_{1}$ space group $\left(n^{\circ} .29\right)$ with two magnesium(II) phthalocyanine molecules (A and B), two nitrate anions and one tetrahydrofurane molecule in general position. The formulation is then $(\mathrm{MgPc})\left(\mathrm{NO}_{3}\right)^{\cdot} 0.5 \mathrm{THF}$, corresponding to $a+1$ oxidation state for each phthalocyanine ring. The phthalocyanine rings adopt a regular geometry, with the magnesium cations lying out of plane (0.600(4) and $0.597(4) \AA$ for $\mathbf{A}$ and B respectively). Each anion is strongly bound to one $\mathrm{Mg}$ (II) cation via one oxygen atom and weakly by another one. Coordination environment around Mg(II) can then be described as intermediate between a pentacoordinated distorted square planar pyramid and a hexacoordinated trigonal prism (molecule A: $\mathrm{Mg}-\mathrm{N}=2.041(6), 2.055(6), 2.055(6)$ and 2.067(6) $\AA, \mathrm{Mg}-\mathrm{O}=2.050(6)$ and 2.841(7) $\AA$; molecule B: $\mathrm{Mg}-\mathrm{N}=2.045(6), 2.048(6), 2.049(6)$ and 2.070(6) $\AA, \mathrm{Mg}-\mathrm{O}=2.073(6)$ and 2.585(7) $\AA$ ). Coordination of the anion to the cation strongly affects the shape of the open-shell species (compare the geometry of the cationic, symmetrical and planar ${ }^{1} \mathrm{MgPc}^{{ }^{+}}$to that of the neutral, unsymmetrical and non-planar $\left.\mathrm{MgPc}\left(\mathrm{NO}_{3}\right)^{\circ}\right)$. Molecules $\mathbf{A}$ and $\mathbf{B}$ are associated along

\footnotetext{
${ }^{1}$ (a) Schramm, C. J.; Scaringe, R. P.; Stojakovic, D. R.; Hoffman, B. R.; Ibers, J. A.; Marks, T. J. J. Am. Chem. Soc. 1980, 102, 6702-6713. (b) Martinsen, J.; Stanton, J. L.; Greene, R. L.; Tanaka, J.; Hoffman, B. M.; Ibers, J. A. J. Am. Chem. Soc. 1985, 107, 6915-6920. (c) Gardberg, A. S.; Deng, K.; Ellis, D. E.; Ibers, J. A. J. Am. Chem. Soc. 2002, 124, 5476-5480.
} 
the $a$ axis to form X-shaped dimers (shortest C••C contacts: 3.130(9), 3.151(9), 3.152(10), 3.156(9), 3.165(9), 3.174(9), 3.183(8) and 3.189(9) $\AA$ ). This motif is commonly observed in phthalocyanine-based radical cation salts, but usually develops in one direction. In our case, due to the presence of bulky ligands on the magnesium cations, one dimensional stacking is prevented in favor of two dimensional packing, as already emphasized by Inabe and $\mathrm{al}^{2}{ }^{2}$ with other axial substituents. Indeed, $\pi$-contacts (shortest C•.•C contacts: 3.371(9), 3.371(10), 3.389(10) and 3.406 $\AA$ for one contact, 3.540(11), 3.558(11), 3.558(10), 3.581(11), 3.581(10) and 3.585(10) A for the other one) are observed between X-dimers in the $(a+b)$ and $(a-b)$ directions, forming a layer of parallel radicals. These layers are then stacked along the $c$ axis.

2 crystallizes in a monoclinic setting in the centrosymmetric $P 2_{1} / c$ space group $\left(n^{\circ}\right.$. 14) with two magnesium(II) phthalocyanine molecules (A and B), two perrhenate anions and three tetrahydrofuran molecules in general position. The formulation is then $(\mathrm{MgPc})\left(\mathrm{ReO}_{4}\right) \cdot 1.5 \mathrm{THF}$, corresponding to $\mathrm{a}+1$ oxidation state for each phthalocyanine ring.

The phthalocyanine rings adopt once again a regular geometry, with the magnesium cations lying out of plane (0.539(3) and 0.534(3) A for A and B respectively). Each anion is bound to one $\mathrm{Mg}(\mathrm{II})$ cation via one oxygen atom, the corresponding Re-O distance being about $0.06 \AA$ longer than the other Re-O bonds. Coordination environment around $\mathrm{Mg}(\mathrm{II})$ can then be described as a pentacoordinated square planar pyramid (molecule A: Mg-N = 2.040(5), 2.041(5), 2.047(5) and 2.048(5) $\AA$, Mg-O = 2.000(4) $\AA$; molecule B: $M g-N=2.035(5), 2.039(5), 2.044(5)$ and 2.045(5) $\AA$, Mg-O = 2.004(4) $\AA$ ). As in 1, A and B molecules associate in X-shaped dimers (shortest C••C contacts: 3.133(7), 3.162(7), 3.169(7), 3.171(7), 3.190(8), 3.190(7) and 3.198(8) A) with bulky perrhenate ligands preventing the formation of one-dimensional stacks of open-shell species.

\footnotetext{
${ }^{2}$ For discussions see: (a) Inabe, T.; Maruyama, Y, Mitsuhashi, T, Synth. Met. 1991, 42(3), 2629-2632. (b) Matsumara, N, Fujita, A.; Nato, T.; Inabe, T. J. Mater. Chem. 2000, 10, 2266-2269. (c) Inabe, T. J. Porphyrins Phthalocyanines 2001, 5, 3-12. (d) Inabe, T.; Asari, T.;Hasegawa, H.; Matsuda, M.; Gacho, E. H.; Matsumara, N.; Takeda, S.; Takeda, K.; Naito, T. Synth. Met. 2003, 133-134, 515-518. (e) Asari, T.; Naito, T.; Inabe, T.; Matsuda, M.; Tajima, H. Chem. Lett. 2004, 33(2), $128-129$.
} 
X-dimers form layers of parallel radicals in the $(b, c)$ plane by the intermediate of short $\pi$ - $\pi$ contacts (shortest C...C contacts: 3.226(7), 3.392(8), and 3.400(7) Å between A molecules, 3.266(7) and 3.376(8) A between B molecules, and 3.386(8) and 3.387(8) Å between A ad B molecules). These layers stack in the a direction, with all macrocycles parallel to each other and short $\mathrm{C} \cdot \bullet \mathrm{C}$ contacts between consecutive layers (3.392 (9) ^̊). 
TCNQ radical anion packings.

$$
\text { (TCNQ) } 2^{\text {n- }} \text { stacking }
$$

Staggered along the long axis $(57 / 71)$

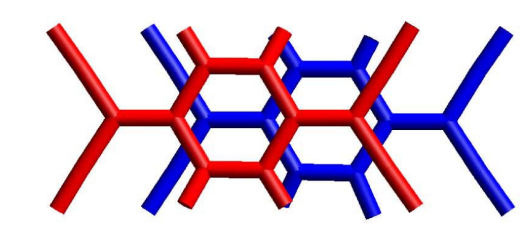

staggered along the short axis $(10 / 71)$

Eclipsed

$(1 / 71)$

Fully staggered along the long axis (2/71)
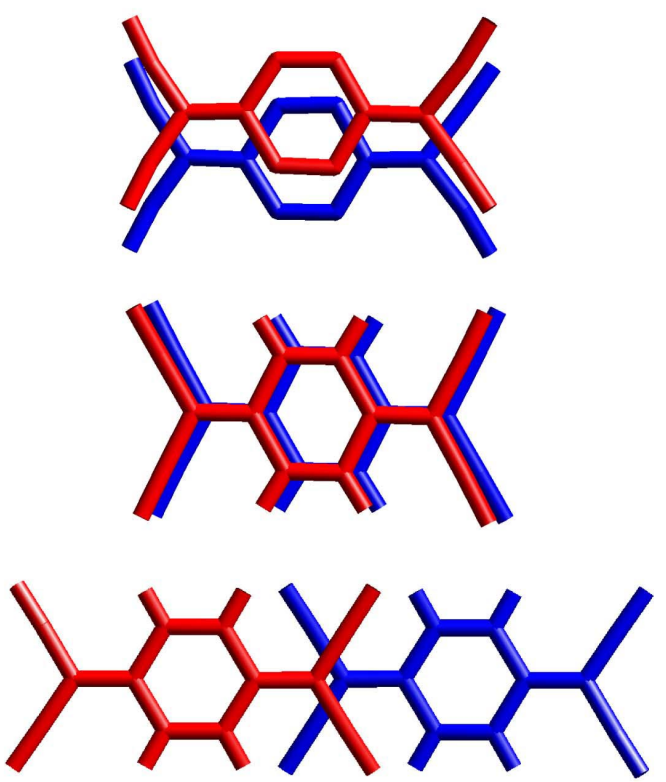

No dimer

$(1 / 71)$

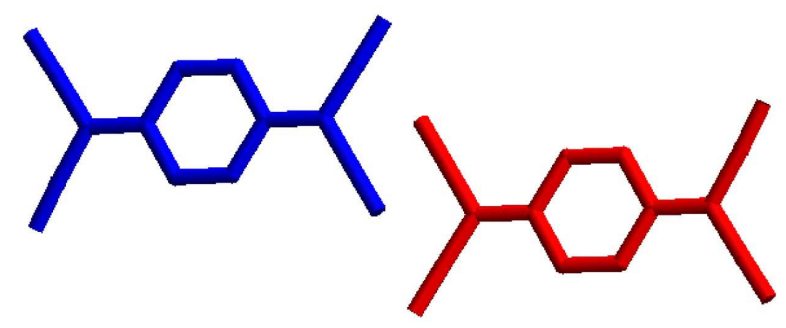




\section{Complete Reference 82.}

Frisch, M. J.; Trucks, G. W.; Schlegel, H. B.; Scuseria, G. E.; Robb, M. A.; Cheeseman, J. R.; Zakrzewski, V. G.; Montgomery, J. A. Jr.; Stratmann, R. E.; Burant, J. C.; Dapprich, S.; Millam, J. M.; Daniels, A. D.; Kudin, K. N.; Strain, M. C.; Farkas, O.; Tomasi, J.; Barone, V.; Cossi, M.; Cammi, R.; Mennucci, B.;

Pomelli, C.; Adamo, C.; Clifford, S.; Ochterski, J.; Petersson, G. A.; Ayala, P. Y.; Cui, Q.; Morokuma, K.; Malick, D. K.; Rabuck, A. D.; Raghavachari, K.; Foresman, J. B.; Cioslowski, J.; Ortiz, J. V.; Baboul, A. G.; Stefanov, B. B.; Liu, G.; Liashenko, A.; Piskorz, P.; Komaromi, I.; Gomperts, R.; Martin, R. L.; Fox, D. J.; Keith, T.; Al-Laham, M. A.; Peng, C. Y.; Nanayakkara, A.; Challacombe, M.; Gill, P. M. W.; Johnson, B.; Chen, W.; Wong, M. W.; Andres, J. L.; Gonzalez, C.; Head-Gordon, M.; Replogle, E. S.; Pople, J. A. Gaussian 98, Revision A.9; Gaussian Inc.: Pittsburgh, PA, 1998.

\section{Definitions for $\mathbf{x}, \mathbf{y}$ and $\theta$ in the fluoranthene and pyrene systems.}
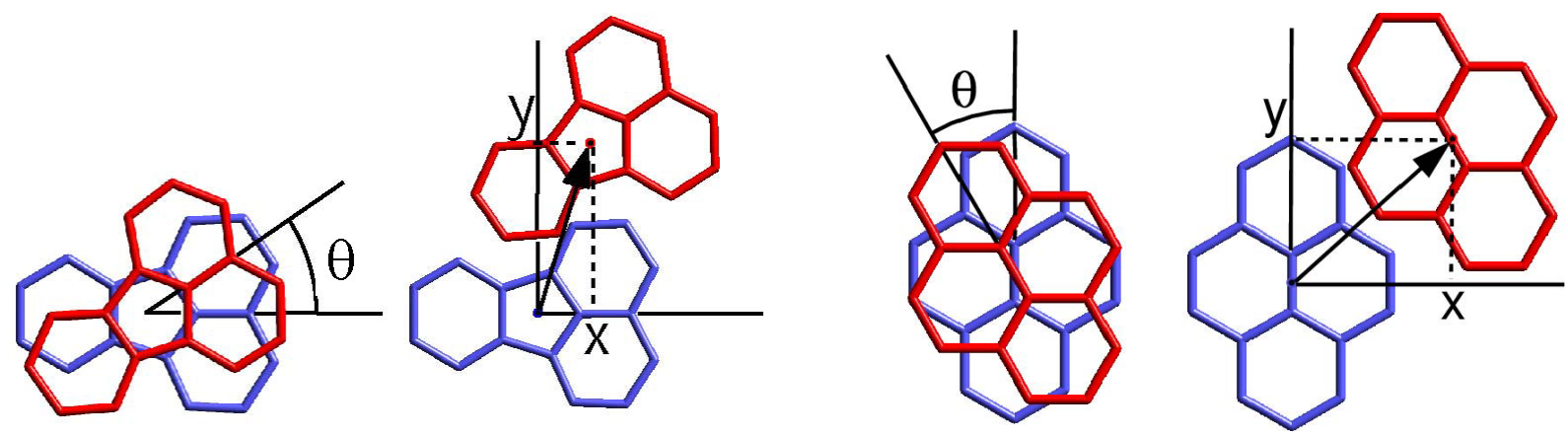

The three parameters used in the energy contour maps for fluoranthene (left) and pyrene (right). $x$ and $y$ : the relative position of the center of 5-member ring of two fluoranthene moieties (left), or the relative position of the center-of-mass of two pyrene moieties (right). $\theta$ : rotation angle of two fluoranthene molecules (left) or two pyrene molecules (right). 